\title{
One patient with two different 18-fluorodeoxyglucose positron emission tomography/computed tomography views
}

\author{
Meryem Ö Özkan ${ }^{1}$, Mine Ş Eren ${ }^{1}$, Sibel Göksel ${ }^{1}$, Yakup Yürekli ${ }^{1}$, Sabri Barutça ${ }^{2}$ \\ 1. Department of Nuclear Medicine, Adnan Menderes University of Medicine School, Aydın, Turkey. 2. Department of \\ Medical Oncology, Adnan Menderes University of Medicine School, Aydın, Turkey.
}

Correspondence: Mine Ş Eren. Address: Department of Nuclear Medicine, Adnan Menderes University of Medicine School, Aydın, Turkey. Email: minesencan67@mynet.com

Received: April 8, 2014

Accepted: June 16, 2014

Online Published: June 26, 2014

DOI: $10.5430 /$ ijdi.v1n2p114

URL: http://dx.doi.org/10.5430/ijdi.v1n2p114

\section{Abstract}

A normoglycemic patient with a diagnosis of laryngeal cancer underwent F-18 Fluorodeoxyglucose Positron Emission Tomography/Computed Tomography (F-18 FDG PET/CT) for staging purposes and two different results were obtained. In the first assessment, F-18 FDG uptake of the primary lesion was very low, but a significant F-18 FDG uptake was noted in the second evaluation.

F-18 FDG PET/CT is used for the diagnosis, staging and re-staging, as well as for the assessment of response to treatment in oncological patients. F-18 FDG uptake is associated with fasting blood glucose levels and satiety. The lower and upper limit of fasting blood glucose is variable between centers.

In this present case report, we want to discuss the factors responsible for not detecting F-18 FDG uptake in the area of primary lesion in a normoglycemic patient with laryngeal cancer.

\section{Keywords}

F-18 FDG, Normoglycemia, Uptake, Insulin

\section{Introduction}

F-18 Fluorodeoxyglucose Positron Emission Tomography/Computed Tomography (F-18 FDG PET/CT) is a commonly used and highly valuable imaging test used for diagnosis, staging, re-staging and evaluation of response to treatment in patients with oncological diseases ${ }^{[1-4]}$. The degree of F-18 FDG uptake by metabolically active cells may depend on various factors. Besides fasting blood glucose levels, the fasting status of the patient carries importance.

In the literature, the recommended range of fasting blood glucose levels during F-18 FDG PET/CT examination is between 140 and 200mg/dL. It has been reported that the lower the fasting blood glucose level is the more significant is the distribution of FDG. Likewise, fasting duration of the patient is also important. In some centers, it is recommended that patients should fast for 4-6 hours before the test, while some centers recommend 12 hours of fasting ${ }^{[5]}$. 


\section{Case presentation}

A vegetative laryngeal mass was detected in the otorhinolaryngological examination of a 64 year old male patient with a complaint of hoarseness. The histopathological report of the biopsy sample taken from the right vocal cord revealed squamous cell carcinoma. F-18 FDG PET/CT examination was requested for staging of the primary tumor.

On the research day, the patient indicated that he had no history of diabetes and he had been fasting for 12 hours.

Before F-18 FDG PET/CT test, the patient's blood glucose level measured using a glucose meter was $74 \mathrm{mg} / \mathrm{dL}$. One hour after injection of $5 \mathrm{mCi}$ (185 Mbq) of F-18 FDG intravenously, a low dose CT scan from vertex to proximal thigh was obtained for anatomic correlation and attenuation correction (Siemens Biograph $20 \mathrm{mCT}$ ). Then, PET images were taken from proximal thigh to the vertex at 2 minute/bed position for 5-6 bed positions. When the F-18 FDG PET/CT images were evaluated, it was observed that there was mild diffuse F-18 FDG uptake in the muscles and in the primary lesion (SUVmax 2.1), and there was marked cardiac activity (see Figure 1). When questioned, the patient stated that he had eaten a little bit. A new date was scheduled for the examination and the patient was recommended to fast for 12 hours before coming.
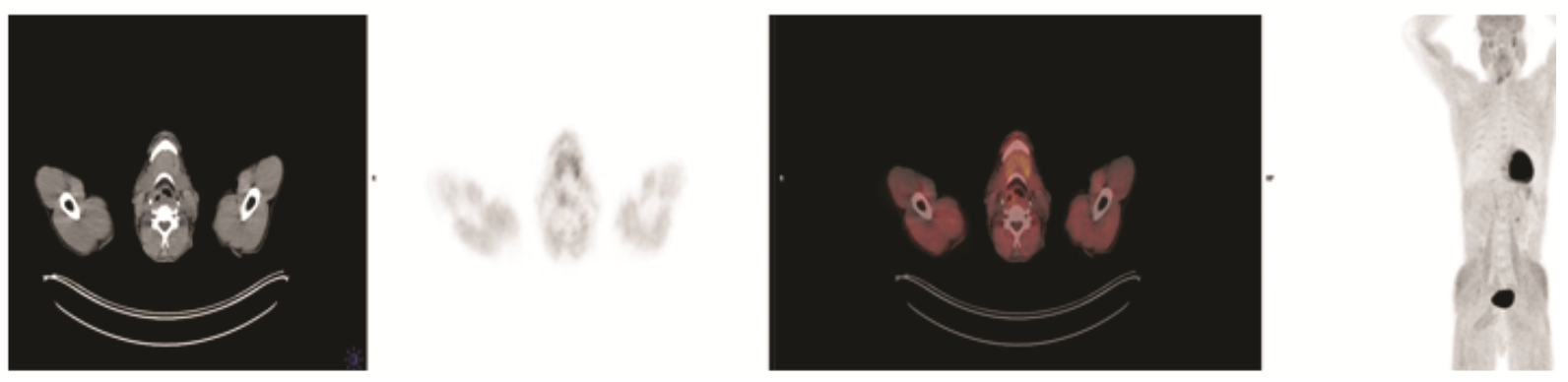

Figure 1. The first scan. F-18 FDG PET/CT image showing F-18 FDG uptake in the muscles and heart; mild FDG uptake was noted in the larynx.

Before the second test, blood glucose level measured with the glucose meter was $77 \mathrm{mg} / \mathrm{dL}$. The patient was given the same dose of F-18 FDG by intravenous injection and a second PET/CT scan was performed using the same protocol. There was a significant F 18 FDG uptake in the primary lesion (SUVmax: 11.4), however, no F-18 FDG uptake was observed in the heart and muscles (see Figure 2).
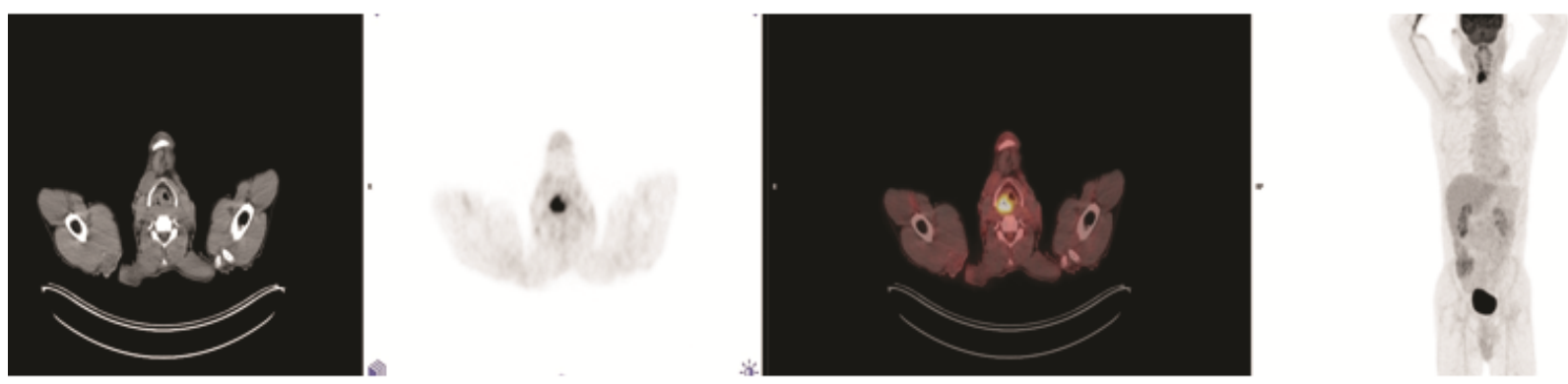

Figure 2. The second scan. F-18 FDG PET/CT image showing F-18 FDG uptake in the larynx.

\section{Discussion}

The high glucose metabolism of tumor cells in comparison to normal cells is the basis of using F-18-FDG for tumor imaging in PET-CT. Increased glycolysis in tumor cells leads to an increase in the number of glucose transporter proteins 
(GLUT), especially GLUT1 and intracellular hexokinase enzyme levels. F-18 FDG, after intravenous administration, uses the same pathways as glucose until a certain step of glucose metabolism. Similar to glucose, F-18 FDG is transported into the tumor cell by insulin dependent GLUT1, and phosphorylated into F-18 FDG-6P by hexokinase enzyme. As F-18 FDG-6P, is not a suitable substrate for glucose-6 phosphate-isomerase, it does not enter the other metabolic reactions of glycolysis, and continues to accumulate in tumor cells as long as it is transported ${ }^{[4,6]}$.

The physiological distribution of F-18 FDG in the body is variable due to different GLUT contents of the tissues. GLUT2 is expressed in the liver and pancreas, whereas GLUT4 is found in the skeletal muscle, heart and adipose tissue ${ }^{[7]}$. In addition, 18 FDG uptake may widely vary depending on exercise, hunger, satiety, fasting blood glucose levels and insulin use ${ }^{[8-10]}$. In addition to patients with high fasting blood glucose levels and those who have eaten food before the examination, F-18 FDG activity is increased in the muscles and heart of patients using insulin ${ }^{[4,10]}$.

It was reported that there was no difference in the liver, muscle and brain SUV values of diabetic patients receiving or not receiving short-acting insulin ${ }^{[11]}$.

In the case presented herein, although there was significant F-18 FDG uptake in the heart and muscle tissue, F-18 FDG uptake was very mild in the primary lesion in the first examination. However, in the repeated scan after prolonged fasting, while there was no FDG uptake in the heart and skeletal muscle, there was significant FDG uptake in the primary lesion. The FDG uptake in the heart and muscles was attributed to the increased postprandial endogenous insulin levels as the blood sugar of our patient was within normal ranges. However, we cannot confirm this situation, as we did not assess the insulin levels of our patient.

F-18 FDG PET/CT tests are often used to assess response to treatment and restaging in oncological cases ${ }^{[4]}$, and scans are obtained according to guidelines on the quality and technique of scanning and patient preparation ${ }^{[5]}$.

In these cases, all PET/CT scans should be performed under the same conditions as the first PET/CT (same fasting period and the same dose) ${ }^{[5,12]}$. The differences in the pre-test preparation may lead to differences in FDG uptake of the lesion, and regression of a lesion may be interpreted as progression and vice versa.

We suggest that assessment of only fasting blood glucose levels is not sufficient in patients undergoing F-18 FDG PET/CT, and insulin (insulin peptide C) levels should also be assessed.

\section{References}

[1] Bruel AV, Maes A, Potter TD et al. Clinical relevance of thyroid fluorodeoxyglucose-whole body positron emission tomography incidentaloma. J Clin Endocrinol Metab. 2002; 87: s1517-s20. http://dx.doi.org/10.1210/jcem.87.4.8371

[2] Yasuda S, Ide M, Fujii HT et al. Application of positron emission tomography imaging to cancer screening. British Journal of Cancer. 2000; 83: s1607-s11. dx.doi.org/10.1054/bjoc.2000.1496

[3] Van Westreenen HL, Westerterp M, Jager PL et al. Synchronous primary neoplasms detected on 18F-FDG PET in staging of patients with esophageal cancer. J Nucl Med. 2005; 46: s1321-s5. http://dx.doi.org/content/46/8/1321.long

[4] Ziessman HA, O’Malley JP, Thrall JH. Oncology: Positron Emission Tomography. In: Thrall JH ed. Nuclear Medicine The Requisites in Radiology 3rd ed. Philadelphia: Mosby Elsevier; 2006. s302-s45.

[5] Boellaard R, O’Doherty MJ, Weber WA et al. FDG PET and PET/CT: EANM procedure guidelines for tumour PET imaging: version 1.0. Eur J Nucl Med Mol Imaging. 2010; 37: s181-s200. http://dx.doi.org/10.1007/s00259-009-1297-4

[6] Haber RS, Weiser KR, Pritsker A et al. GLUT1 glucose transporter expression in benign and malignant thyroid nodules. Thyroid. 1997; 7: s363-s67 [abstract]. PMid: 9226204. Available from: http://www.liebertonline.com/thy

[7] Kaneta T, Hakamatsuka T, Takanami K et.al. Evaluation of the relationship between physiological FDG uptake in the heart and age, blood glucose level, fasting period, and hospitalization. Ann Nucl Med. 2006; 20: s203-s8 [abstract]. Available from: http://link.springer.com/journal/12149 
[8] Vrieze A, Schopman JE, Admiraal WM et al. Fasting and postprandial activity of brown adipose tissue in healthy men. J Nucl Med. 2012; 53: 1407-10. http://dx.doi.org/10.2967/jnumed.111.100701

[9] Reinking MF, Osman MM. Prospective evaluation of physiologic uptake detected with true whole-body 18F-FDG PET/CT in healthy subjects. J Nucl Med Technol. 2009; 37: 31-7. http://dx.doi.org/ 10.2967/jnmt.108.055004

[10] Kreissi MC, Stout DB, Wong KP et al. Influence of dietary state and insulin on myocardial, skeletal muscle and brain $\left[{ }^{18} \mathrm{~F}\right]$-fluorodeoxyglucose kinetics in mice. EJNMMI Research. 2011; 1: s8. http://dx.doi.org/10.1186/2191-219X-1-8.

[11] Song HS, Yoon JK, Lee SJ et al. Ultrashort-acting insulin may improve on 18F-FDG-PET/CT image quality in patients with uncontrolled diabetic mellitus. Nuclear Medicine Communications. 2013, 34: s527-s32. http://dx.doi.org/10.1097/MNM.0b013e32836066a2

[12] Shankar LK, Hoffman JM, Bacharach S et al. Consensus Recommendations for the Use of 18F-FDG PET as an Indicator of Therapeutic Response in Patients in National Cancer Institute Trials. 2006; 46: s1059-s1066.

http://jnm.snmjournals.org/content/47/6/1059 